\section{Long term efficacy and safety of a combined low and high molecular weight hyaluronic acid in the treatment of osteoarthritis of the knee}

\author{
Robert J. Petrella,1,2,3 Joseph Decaria,,1,2 \\ Michael J. Petrella' \\ 'Lawson Health Research Institute, \\ University of Western Ontario, London, \\ Canada; ${ }^{2}$ School of Kinesiology, \\ University of Western Ontario, London; \\ ${ }^{3}$ Faculty of Medicine and Dentistry, \\ University of Western Ontario, Canada
}

\section{Abstract}

The primary objective was to determine the efficacy of intraarticular combined hyaluronic acid versus placebo in patients with grade 1-3 medial compartment osteoarthritis of the knee as evaluated through the self-paced $40 \mathrm{~m}$ walking pain visual analog scale (VAS) at week 16 , 52 and 104. Secondary objectives included pain at rest: a $10 \mathrm{~cm}$ VAS, patient global satisfaction using a 5-point numerical scale, consumption of concomitant medications, patients with $<45$ mm pain at followup 52 and 104 weeks. Safety was determined through the number of recorded adverse events. The study was designed as a prospective, randomized, double-blind, placebo controlled and comparative study. 200 patients were randomized in a 1:1 ratio to one of four treatment groups, to receive 3 weekly intra-articular injections of either: DMW (combined HA of different molecular weight and concentrations); HMW (high molecular weight HA); LMW (low molecular weight HA); PL (placebo, saline). Patients were assessed baseline and at week 16, 52, 104. Analyses were conducted using sigma stat (SPSS Inc., Chicago, Illinois) and Microsoft Excel (Microsoft Corp, Redmond, Washington). Significance was established at $\mathrm{P}<0.05$. Analysis of variance with repeated measures and $\chi^{2}$ tests were used to test for differences from baseline characteristics of the group among the primary and secondary outcomes at each injection series interval. At 16,52 and 104 weeks respectively, walking VAS pain was significantly improved in all treatment groups vs. Placebo: DMW (89.3\%, $\mathrm{P}<0.001 ; 87.4 \%$, $\mathrm{P}<0.001 ; 88.1 \%, \quad \mathrm{P}<0.001) ;$ LMW $(81.3 \%$, $\mathrm{P}<0.001 ; 78.2 \%, \mathrm{P}<0.001 ; 77.0 \%, \mathrm{P}<0.001)$ and HMW (79.1\%, $\mathrm{P}<0.001 ; 81.1 \%, \mathrm{P}<0.001 ; 79.4 \%$, $\mathrm{P}<0.001)$. At 52 weeks, 8 patients in DMW group had resting VAS $<45 \mathrm{~mm}$. DMW had lower (62 mm, P <0.001) compared to LMW $(76 \mathrm{~mm})$ and HMW $(88 \mathrm{~mm})$ VAS at rest.
Similar differences were observed for walking VAS 39, 41 and 43 (DMW, LMW, HMW) received repeat injections. At 104 weeks, these differences were similar. DMW and LMW had no reported adverse events; HMW had 2 local reactions at 52 weeks and 1 at 104 weeks. There were no serious adverse events. Non- serious adverse events included pain and local swelling at the injection site (21\%), erythema at the injection site (12\%) and stiffness in the index knee (7\%). Intra-articular hyaluronic acid injections using any of low, high or combined MW were highly effective in improving resting and moreso, walking pain in patients with osteoarthritis of the knee. Greater improvement in both rest and activity outcomes in patients who received the DMW product, with concomitantly greater patient satisfaction and fewer use of concomitant therapeutic modalities at 16, 52 and 104 weeks suggest that combining a range of MW hyaluronic acid may be advantageous long term, particularly among active osteoarthriris patients.

\section{Introduction}

Osteoarthritis (OA) is the most common form of arthritis in the Western Population. Osteoarthritis of the knee, the principle large joint to be effected, results in disabling symptoms in an estimated 10\% of patients older than 55 years. ${ }^{1}$ According to the World Health Organization (WHO), knee OA is likely to become the fourth most important cause of disability in women and eight most important in men. There is a great burden on health due to both morbidity and costs.

Guidelines have been drawn up by the American College of Rheumatology (ACR), and the European League against Rheumatism (EULAR), for management of knee 0A. Pain killers and non steroidal anti inflammatory drugs (NSAIDs) are the mainstay treatment. ${ }^{2}$ NSAIDs have severe gastro-intestinal side effects, precluding their use for treatment in the elderly population, the population group it most affects. NSAIDs now hold a black box warning now and doctors should make patients aware of the potential side effects. Non-pharmacological interventions such as education, exercise, life style changes, are not really popular among patients, and adherence as such to these is poor.

Intra-articular injection of hyaluronic acid (HA) is recommended and increasingly used in alleviating symptoms. HA is an unbranched, high molecular weight polysaccharide distributed throughout the body, especially as a major component of the synovial fluid and of cartilage. The primary role of the HA in synovial fluid and cartilage is to maintain the visco-elastic structural and functional charac-
Correspondence: Robert J. Petrella, Lawson Health Research Institute, 801 Commissioners Road, London, 0n N6C5J1 Canada.

E-mail: petrella@uwo.ca

Key words: hyaluronic acid, knee osteoarthritis.

Received for publication: 7 January 2011.

Accepted for publication: 1 March 2011.

This work is licensed under a Creative Commons Attribution 3.0 License (by-nc 3.0).

(C) Copyright R.J. Petrella et al., 2011

Licensee PAGEPress, Italy

Rheumatology Reports 2011; 3:e4

doi:10.4081/rr.2011.e4

teristics of the articular matrix. 0steoarthritis is the result of mechanical and biological events that destabilize the normal degradations synthesis of articular cartilage ${ }^{3}$ and is characterized by a decrease in the concentration and molecular weight of $\mathrm{HA}$, which in turn may lead to the hallmark signs of pain and loss of function in weight-bearing joints such as the knee. ${ }^{2}$ Hence, intra-articular viscosupplementation with HA may restore the concentration and molecular weight characteristics in the articular matrix, resulting in improved pain control and function. ${ }^{3}$

Intra-articular HA is indicated currently for use in patients who may not have responded to a program of non-pharmacological therapy and pain control with analgesics including acetaminophen. ${ }^{4}$ Clinical trials of intra-articular HA preparations have shown pain relief significantly greater than those who injected with placebo $^{5-8}$ and comparable or superior to intraarticular glucocorticoids. ${ }^{9-10}$ Although pain relief is achieved more slowly with HA preparations than with intra-articular glucocorticoid injections, the effect may last considerably longer. Similarly, intra-articular HA has shown comparable improvement in pain with oral anti-inflammatory preparations. ${ }^{8}$ Recent metanalyses have shown effect sizes of intra-articular corticosteroid and Sodium Hyaluronate of variable molecular weight to be similar ${ }^{10,11}$ hence, physicians have further support to consider the attributes of HA for treatment along the continuum from early to advanced

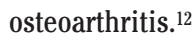

Several HA compounds are currently utilized world-wide by clinicians which differ in molecular weight composition, dosing regimens and claims of efficacy. Specifically, it is unclear whether differences in efficacy are found among products ${ }^{13-15}$ while patients receive specific products without any objective criteria for a given choice. It has been described that differences in HA molecular weight and concentrations in the synovial fluid occur among 
adults with a shift in the elastic-viscous ratio in osteoarthritis that is consistent with the degree of severity and character of symptoms. ${ }^{3}$ The knee in dynamic motion requires elastic composition at optimal molecular weight (MW) in balance with viscous needs. For example, high frequency loading through synovial fluid is dissipated through a dynamic change in Sodium Hyaluronate toward more elastic modulus compared to more viscous properties when the load to Sodium Hyaluronate is of low frequency. ${ }^{3}$ While a given HA product has a limited range of molecular weight typically low, medium or high, no product has been designed to provide a complement of composition that mimics the needs of the active osteoarthritic knee joint. These attributes may promote a more beneficial rheological environment in the osteoarthritic joint. ${ }^{16}$

The purpose of this study was to evaluate the long-term clinical outcomes of pain at rest and following walking activity as well as adverse events, the use of concomitant therapeutic modalities and patient satisfaction following randomization to one of intra-articular viscosupplementation with a lower (500-1000 $\mathrm{kDa}$ ), higher (6 million $\mathrm{kDa}$ ) or combined lower and higher MW (DMW) sodium hyaluronate in osteoarthritis of the knee or placebo.

\section{Materials and Methods}

The primary objective of this study was to determine the efficacy and safety of intraarticular combined molecular weight hyaluronic acid in knee osteoarthritis as evaluated through the self-paced 40-m walking pain visual analog scale (VAS) at week 16, 52 and 104. The secondary endpoints included: pain at rest. A $10 \mathrm{~cm}$ VAS, patient global satisfaction using a 5-point numerical scale, consumption of concomitant medications, number of patients with $<45 \mathrm{~mm}$ pain at followup 52 and 104 weeks, and safety through the number of recorded adverse events. This was a singlecenter, randomized, double-blind, placebo-controlled study. Two hundred eligible consented patients were randomized into four cohorts: Active treatment 1 (DMW); Active treatment 2 (LMW); Active treatment 3 (HMW); Placebo (saline).

Randomization was done using a computergenerated random number table. Physicians and patients were blinded to assignment (injection syringes were covered to conceal any details of the product or volume).

Patients received intra-articular injection once weekly for three weeks. Patients were followed up at week 16, 52 and 104. Study flow chart is illustrated in Table 1.
Patient inclusion criteria included: clinical diagnosis of knee osteoarthritis based on unilateral knee pain and disability; Grade 1-3 medial compartment osteoarthritis previously confirmed through radiographic evidence; VAS of non-weight bearing rest pain score of at least 45 out of $100 \mathrm{~mm}$; willingness and qualified for intra-articular injections; available for the duration of the study including treatment and follow-up (16 weeks); capable and willing to provide informed consent. Exclusion criteria included: unwilling or unable to give written informed consent or unable to comply with the protocol; pregnant or lactating women; end stage of osteoarthritis; contra-indication to intra-articular injection or known hypersensitivity to Sodium Hyaluronate; planned surgical procedure to the study knee during the study period; currently receiving intra-articular investigational drugs; current receiving or having received intra-articular injection of corticosteroids therapy within less than six months; currently receiving or receiving intraarticular injection of HA therapy within less than six months; dosage of glucosamine and/or chondroitin sulfate, and/or NSAIDs that has been stable over the preceding three months with the dosage remaining constant during the study; active skin disease or infection in the area of the injection site; significant venous or lymphatic stasis present in the legs; any condition/disease which in the opinion of the investigator could interfere with patient compliance and/ or interfere with the interpretation of the treatment results. All concomitant medications were recorded and any intra-articular injection treatment other than study medication for this protocol was prohibited during the course of this study. No new treatment with known or expected therapeutic effect on osteoarthritis was initiated during the study period. The following medications were prohibited in the absence of appropriate wash out periods: intraarticular injection of steroids into the study joint within three months prior to screening; intra-articular injection of HA into the study joint within three months prior to screening; systemic steroids within the last three months prior to screening; anticoagulants except for acetylsalicylic acid up to $325 \mathrm{mg}$ per day.

\section{Study population}

225 patients were assessed for eligibility. 25 patients in total were excluded for not meeting the inclusion criteria $(n=13)$ and refusal to participate $(n=12) .200$ eligible patients were randomized and received treatment. 10 patients $\quad($ Placebo $=3 ; \quad \mathrm{DMW}=2 ; \quad \mathrm{LMW}=1$; HMW =4) dropped out for non-study related reasons. In accordance with the Declaration of Helsinki principle, a patient could withdraw from the study at any time and for any reason.

\section{Treatments}

Eligible patients were randomized and received one of the following treatments based on random assignment: DMW (combined HA of different molecular weight and concentrations); HMW (high molecular weight HA); LMW (low molecular weight HA); PL (placebo, saline). Intra-articular injections were given on a weekly basis for three weeks. Injections were performed utilizing sterile technique. Injections were performed with blinded syringes affixed to a 25-27-gauge, $13 / 4$ inch needle. Skin was prepped using betadine $1 \%$. No anesthetic was

Table 1. Schedule of time and events.

\begin{tabular}{cccccc} 
Baseline & \multicolumn{2}{c}{ Treatment } & \multicolumn{2}{c}{ Follow-up } \\
Procedure & $\begin{array}{c}\text { within } 30 \text { days of } \\
\text { treatment }\end{array}$ & W1 W2 & W3 & W16 W52 W104 & W5
\end{tabular}

$\begin{array}{ll}\text { Informed consent } & X^{1} \\ \text { Medical history } & X^{1}\end{array}$

\begin{tabular}{llllllll} 
Medical history & $X^{1}$ & & & & & & \\
\hline Physical examination & $X^{1}$ & $X$ & & & & & \\
Inclusion/ exclusion & $X^{1}$ & $X$ & & & & & \\
\hline Pregnancy test (if applicable) & X1 & & & & & & \\
Index knee & $X^{1}$ & $X$ & & & & & \\
\hline Knee X-ray (if necessary) & $X^{1}$ & & & & & & \\
Vital signs & $X^{1}$ & $X$ & & & & & \\
\hline 12-lead EKG & $X^{1}$ & & & & & & \\
Blood and urine sample & $X^{1}$ & & & & & & \\
\hline Self paced 40-m walking pain VAS & $X$ & $X$ & $X$ & $X$ & $X$ & $X$ & $X$ \\
Seated rest pain VAS & $X$ & $X$ & $X$ & $X$ & $X$ & $X$ & $X$ \\
\hline Patient global satisfaction & $X$ & $X$ & $X$ & $X$ & $X$ & $X$ & $X$ \\
Intra-articular injection & & $X$ & $X$ & $X$ & & & \\
\hline Pain $<45$ mm & & & & & & $X$ & $X$ \\
Concomitant medications & $X$ & $X$ & $X$ & $X$ & $X$ & $X$ & $X$ \\
\hline Adverse events & & $X$ & $X$ & $X$ & $X$ & $X$ & $X$ \\
\hline
\end{tabular}


used either topically or intra-articularly. Intraarticular injections will be delivered by the study physician using a standard medial approach: after palpation of the superior lateral aspect of the patella, insert the needle into the space between the patella and femur parallel to the inferior border of the patella.

\section{Study materials}

Combined HA (DMW): the DMW preparation consists of $0.7 \mathrm{~mL}$ of sterile $2.2 \%$ LMW (0.58- $0.78 \times 10^{6}$ Daltons) sodium hyaluronate and $0.7 \mathrm{~mL}$ of sterile $1 \%$ HMW $\left(1.2-2.0 \times 10^{6}\right.$ Daltons) sodium hyaluronate. The two different HAs was separated by a Debiopass stopper within a pre-filled $3 \mathrm{ml}$ Lsterile syringe and injected sequentially into the knee joint.

Low molecular weight HA (LMW): Sodium hyaluronate of $500-730 \mathrm{KDa}, 20 \mathrm{mg} / 2 \mathrm{~mL}$ in a prefilled syringe. High molecular weight HA (HMW): Sodium hyaluronate of $6000 \mathrm{KDa}$, $16 \mathrm{mg} / 2 \mathrm{~mL}$ in a prefilled syringe. Placebo: Normal saline $2 \mathrm{~mL}$ in a prefilled syringe. Overall concomitant medications use at baseline for osteoarthritis included acetaminophen (62\%), NSAID/COX-2 (60\%), and nutraceuticals (38\%). Thirteen percent used physical therapy and/or bracing.

\section{Performance measures}

Visual analog scale. This scale consists of a line that is $10 \mathrm{~cm}$ in length and the line is anchored by two extremes of pain (Figure 1).

\section{Adverse events}

All adverse events (AE) were recorded during the trial period. The investigator and co-investigators had to enquire about subjective or objective AEs including abnormal laboratory findings. All AEs were assessed for intensity and relationship to the study treatment and were followed up to resolution or to the end of the study period. The intensity of AEs was graded on a 3-point scale: mild, moderate, severe.

\section{Statistical analysis}

Analysis of variance with repeated measures and $\chi^{2}$ test were used to test for differences from baseline characteristics of the group among the primary and secondary outcomes at each injection series interval. Analyses were conducted using sigma stat (SPSS Inc., Chicago, Illinois, USA) and Microsoft Excel (Microsoft Corp, Redmond, Washington, USA). Significance was established at $\mathrm{P}<0.05$ and included 95\% Confidence Intervals (CI). The sample size was determined to allow the detection of a $20 \mathrm{~mm}$ difference in weight-bearing VAS at W16 assuming a standard deviation 10 $\mathrm{mm}$ of the mean distribution, an a of $5 \%$, and a b level of $10 \%$, giving a statistical power of $90 \%$. With a potential dropout rate of $20 \%$, we estimated a sample size of 225 patients.

\section{Results}

Subject disposition is illustrated in Figure 2.

\section{Demographic and baseline charac- teristics}

The study population did not differ in baseline characteristics from the total referral group over the recruitment period. Study population and total referral baseline demographics are given in Table 2.

1. At rest in the clinical chair: The Visual Analog Scale (VAS 0-10 cm)

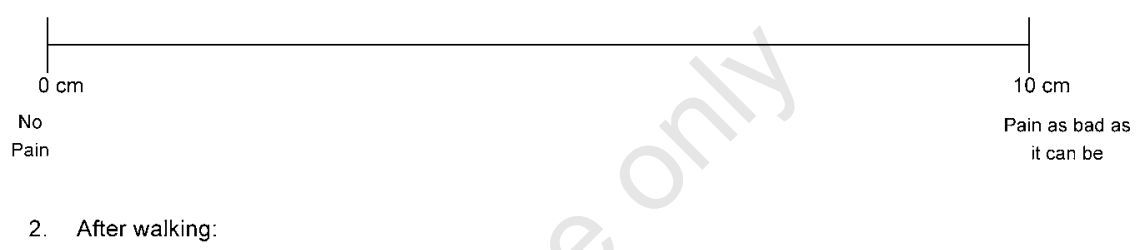

Patient Global Satisfaction

The physician performed a global assessment of the knee involved in the study based on a five point categorical scale.

\begin{tabular}{|c|c|c|c|c|}
\hline $\begin{array}{c}\text { Markedly } \\
\text { worse }\end{array}$ & Worse & No change & Improved & $\begin{array}{c}\text { Nrkedly } \\
\text { inoroved }\end{array}$ \\
\hline & & & & \\
\hline
\end{tabular}

Figure 1. Visual analog scale. This scale consists of a line that is $10 \mathrm{~cm}$ in length and the line is anchored by two extremes of pain.

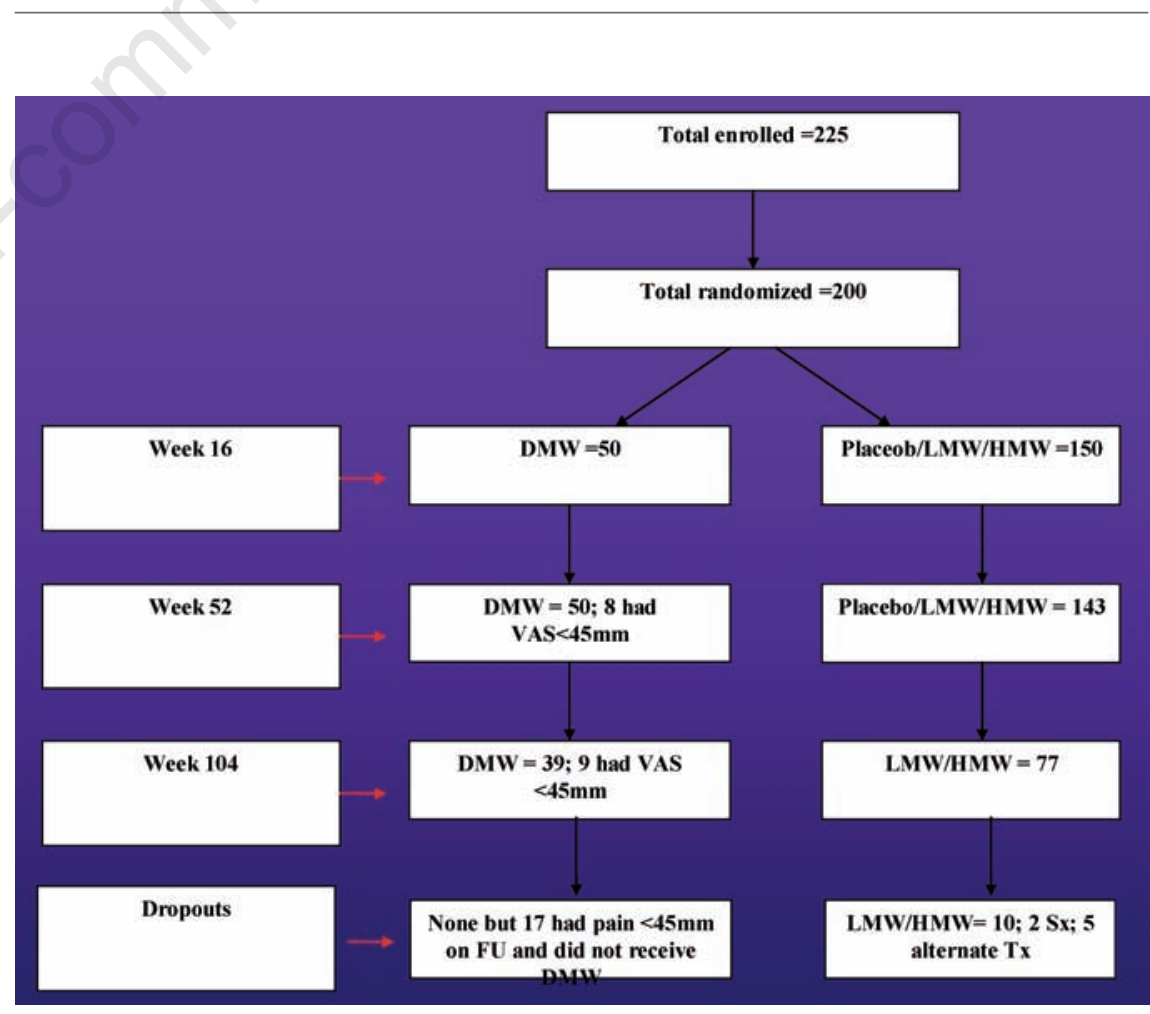

Figure 2. Study flow chart.

\section{Subject disposition}

Greater improvement in patients who received the DMW product was achieved by the second injection and was persistent to 16 weeks. At 52 weeks, 8 patients in DMW had VAS $<45 \mathrm{~mm}$ and were not given repeat injections. DMW walking pain reduction was significantly greater than either of the LMW or HMW groups. 39, 41 and 43 patients received repeat DMW, LMW and HMW at week 52. Placebo patients were provided any therapy option of their choice (LMW or HMW). Those who did not receive injection in the active treatment 
groups dropped out to receive alternate oral medication $(\mathrm{N}=5)$ or opted for surgical intervention $(\mathrm{N}=2)$. At 104 weeks, 9 patients had VAS pain $<45 \mathrm{~mm}$ in the DMW while all in the LMW or HMW groups was $>45 \mathrm{~mm}$. Only 3 patients in the HMW group did not return for followup (2 had moved away and one refused to return). Again, those who received DMW had significantly greater walking pain reduction compared to the other 2 groups. Similar trends in secondary outcomes were also observed at all time points. Combination of HA of lower and higher ranges of MW with low and high concentrations, may provide patients with a more physiologically dynamic HA viscosupplementation and hence a more responsive synovial rheology that improves pain and function in their osteoarthritic knee.

At 16, 52 and 104 weeks respectively, the change in walking VAS pain significantly improved from baseline in all three active treatment groups: DMW $(89.3 \%, \mathrm{P}<0.001$; $87.4 \%, \quad \mathrm{P}<0.001 ; 88.1 \%, \quad \mathrm{P}<0.001) ; \quad$ LMW (81.3\%, $\mathrm{P}<0.001 ; 78.2 \%, \mathrm{P}<0.001 ; 77.0 \%, \mathrm{P}$ $<0.001)$ and HMW (79.1\%, $\mathrm{P}<0.001 ; 81.1 \%$, $\mathrm{P}<0.001 ; 79.4 \%, \mathrm{P}<0.001)$. At 52 weeks, 8 patients in DMW group had resting VAS $<45$ $\mathrm{mm}$. DMW had lower $(62 \mathrm{~mm}, \mathrm{P}<0.001)$ compared to LMW $(76 \mathrm{~mm})$ and HMW $(88 \mathrm{~mm})$ VAS at rest. Similar differences were observed for walking VAS. 39, 41 and 43 (DMW, LMW, HMW) received repeat injections. At 104 weeks, these differences were similar.

Patients in the DMW group had significantly greater improvement at 16, 52 and 104 weeks $(\mathrm{P}<0.001)$ compared to the other active treatment groups which did not differ from each other.

Rest VAS pain was significantly decreased in all 3 active treatment groups from baseline at 16,52 and 104 weeks, however, there was no significant difference among groups (Figure 3).

\section{Safety}

In this study, there were no serious adverse events up to 104 weeks. Non-serious adverse events were associated with the injection procedure and included pain and local swelling at the injection site (21\%), erythema at the injection site (12\%) and stiffness in the index knee (7\%). By 104 weeks, only 2 patients opted for surgical intervention and only 5 opted for an alternate therapy. There was no difference between groups for any of these reported events. None of the adverse events resulted in delay in study procedures.

Intra-articular injection of Sodium Hyaluronate is in general well tolerated. Ten subjects (Placebo=3; DMW $=2 ; \quad \mathrm{LMW}=1$; HMW=4) dropped out for non-study related reasons during the first 16 weeks and only 9 dropped out (opted for alternate therapy) from 16 to 104 weeks. There were no serious adverse events. Global satisfaction was signifi-

Table 2. Baseline characteristics of the groups.

\begin{tabular}{|c|c|c|c|c|}
\hline $\begin{array}{c}\text { Variable } \\
N=50\end{array}$ & $\begin{array}{c}\begin{array}{c}\text { Placebo } \\
N=50\end{array}\end{array}$ & $\begin{array}{l}\mathrm{DMW} \\
\mathrm{N}=50\end{array}$ & $\begin{array}{l}\mathrm{LMW} \\
\mathrm{N}=50\end{array}$ & HIMW \\
\hline Age (years) & $71 \pm 8$ & $68 \pm 6$ & $69 \pm 5$ & $71 \pm 9$ \\
\hline $\begin{array}{l}\text { Gender } \\
\text { Female, n } \\
\text { Male, n }\end{array}$ & $\begin{array}{l}30 \\
20\end{array}$ & $\begin{array}{c}8 \\
22\end{array}$ & $\begin{array}{l}27 \\
23\end{array}$ & $\begin{array}{l}29 \\
21\end{array}$ \\
\hline BMI $\left(\mathrm{Kg} / \mathrm{m}^{2}\right)$ & $27.2 \pm 2.1$ & $26.9 \pm 3.0$ & $27.3 \pm 2.1$ & $26.7 \pm 2.6$ \\
\hline Years of OA symptoms & $7.4 \pm 4.1$ & $6.9 \pm 5.0$ & $8.1 \pm 6.0$ & $9.1 \pm 6.7$ \\
\hline Grade knee OA $(1$ or $2, n)$ & 39 & 41 & 41 & 38 \\
\hline Use of concomitant OA therapies (n) & $3 \pm 1$ & $2 \pm 1$ & $3 \pm 2$ & $2 \pm 1$ \\
\hline Prior use of HA product (n) & 10 & 7 & 9 & 7 \\
\hline
\end{tabular}

Values mean \pm SD.

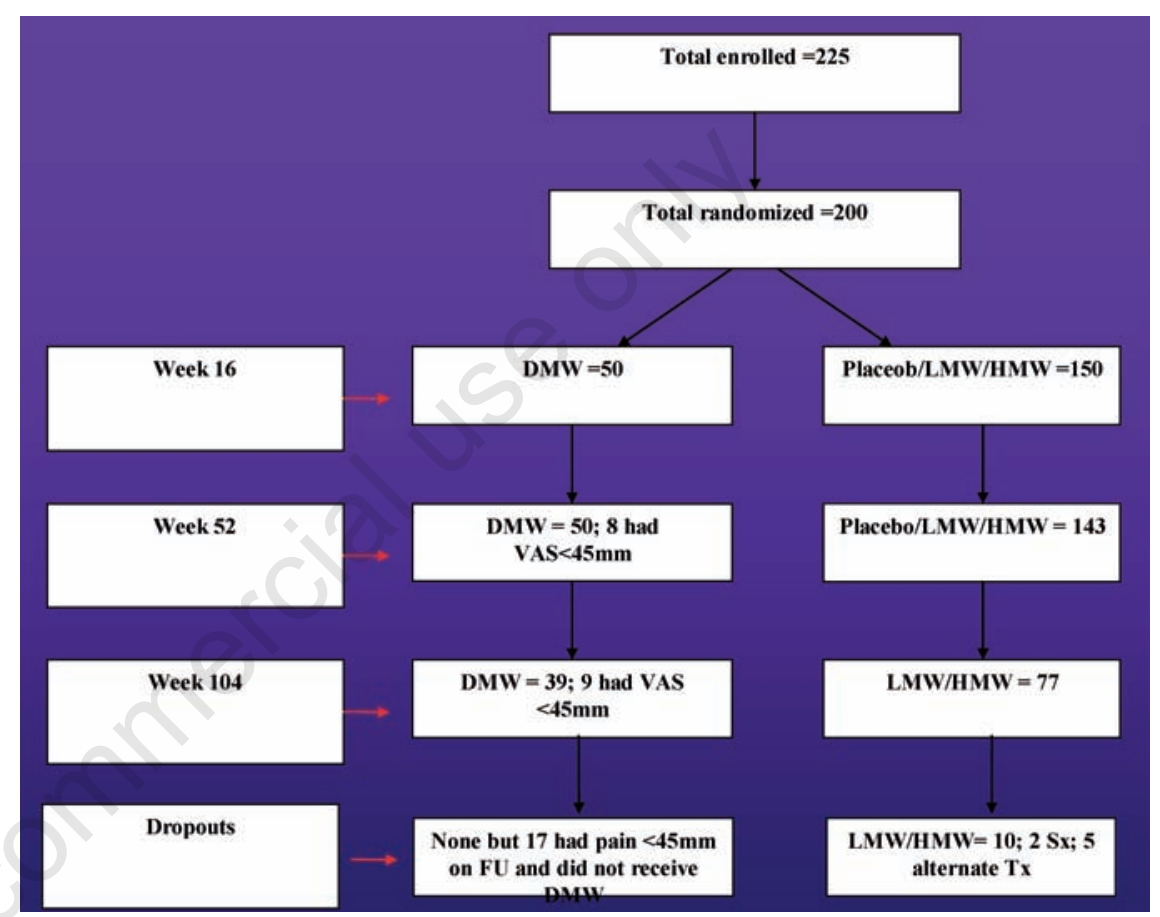

Figure 3. Resting visual analog scale among treatment groups.

cantly higher for the DMW group compared to the other groups at 16, 52 and 104 weeks $(\mathrm{P}<0.005)$. Further, fewer concomitant treatments (ie PT, acupuncture) were utilized by those who received DMW compared to the other treatments at all followup periods.

\section{Discussion}

This study observed a significant improvement in pain at rest and with activity among patients with osteoarthritis of the knee randomized to one of LMW, HMW or combined (DMW) HA interventions. However, patients to whom the DMW HA was administered showed significantly lower activity-related pain, with fewer adverse events (low overall for all 3 treatments) and fewer concomitant therapeutic modalities after 16, 52 and 104 weeks compared to either LMW or HMW-only treatments. DMW patients achieved maximum improvement in VAS pain following the second injection which was greater than the other treatments at any time point. Further, patients in the DMW group showed significantly greater scores of satisfaction with their treatment at 16, 52 and 104 weeks compared to the other two groups. These findings suggest combination of HA of lower and higher ranges of MW may provide patients with a more physiologically dynamic HA viscosupplementation and hence a more responsive synovial rheology that improves pain and function in their osteoarthritic knee. Importantly and in contrast to many other currently approved products, we followed patients for up to 104 weeks 
showing both the long-term efficacy and safety of these products.

DMW is in a dual chamber syringe containing Sodium Hyaluronate of both high and low molecular weights at low and high concentrations. Sodium Hyaluronate of varying molecular weights have been compared and yet there has been no conclusive evidence to support the superiority of any of them. Sodium Hyaluronate concentration may play a more definite role and some published evidences suggest a direct effect over the viscosity of the synovial fluid as well as helping boundary lubrication and thin film lubrication, both mechanisms which are implicated in the joint. ${ }^{17} \mathrm{DMW}$, thanks to its sequential injection of Sodium Hyaluronate of different molecular weight and concentration contributes to the synovial fluid's characteristics by increasing its elastic deformation when under load and hydrodynamic effect which forces contacting surfaces apart when the pressure of the load is deforming them..$^{18}$ This attributes to a higher capability of the synovial fluid in the protection of the joint and in weight bearing and moving. ${ }^{19}$

Previous reports have described the efficacy of Sodium Hyaluronate in patients with osteoarthritis of the knee..$^{6-9}$ Our findings in all three groups support Sodium Hyaluronate as effective in improving symptoms and function in osteoarthritis of the knee with few adverse events. However, it is also interesting to test the hypothesis that synovial fluid, being a dynamic component of knee adaptation to loading of both high and low frequency, may, when compromised by the presence of osteoarthritis, require tailored viscosupplementation of Sodium Hyaluronate that covers both the low and high ranges of MW needs. This concept is not new. Balasz and Denlinger ${ }^{3}$ described a trend toward a progressive loss of a balance between elastic and viscous synovial fluid composition with presence (and progression) of osteoarthritis of the knee while Greenberg et al. ${ }^{16}$ have more recently described the biochemical benefits of two different HA's in a co-culture model of OA. Hence, symptoms of pain with activity, of differing degree in different patients that changes in severity in time, may be related to this alteration of synovial fluid rheology. Viscosupplementation with $\mathrm{HA}$ is a therapeutic attempt to provide temporary relief of osteoarthritis symptoms based on these analogies. However, available products vary in the ranges of MW they contain - primarily being in lower or higher MW. ${ }^{14}$ Hence, it seems reasonable to postulate that current viscosupplementation may fall short in providing patients with the combination of HA MW ranges they need to control their symptoms.

The key findings in this study were the significant improvement in pain and function with less use of concomitant therapies among patients randomized to DMW HA compared to either LMW or HMW alone, and the long term efficacy and safety. Further, improvement was achieved with only 2 injections of DMW suggesting the benefit of combining two MWs may provide effects sooner and to a greater degree than with a HMW or LMW products alone. Limitations include the absence of longer term data in terms of patient efficacy as well as comparison of multiple combinations of MW ranges and concentration of $\mathrm{HA}$.

In this study, two commercially available HA products with standard dosing and injection regimen were used for the control groups. However, it is possible that alternate dosing regimens, perhaps utilizing alternate molecular weight and concentration of HA could further impact these findings (including longer duration of effects) and require future investigation.

Sodium Hyaluronate injections were highly satisfactory to patients with each HA series and included a very low rate of local adverse events over a very long-term followup. This supports previous reports that HA treatment of osteoarthritis of the knee is a safe, effective therapeutic option. Findings of this study suggest that alteration of MW range may further improve outcomes in these patients.

Adverse events with Sodium Hyaluronate reported in the literature are transient pain at the injection site, knee swelling/effusion. Also, rarely reported were local skin reaction (rash, ecchymosis), pruritus, headache, and fever. Adverse event rate was reported varies from 0 to $4 \%$ per injection in controlled trials, to $1.8 \%$ per Synvisc injection and $0.9 \%$ per LMW HA injection in a randomized, double blind comparison to $1.48 \%$ and $1.32 \%$ with the first and the second injection series for pain and swelling at injection site. ${ }^{19-20}$ The symptoms were mild and resolved in a short time. In some cases the symptoms were extensive. The adverse events experienced in this study were similar or less than those reported previously.

\section{Conclusions}

Greater improvement in patients who received the DMW product was achieved by the second injection persistent to 104 weeks. Combination of Sodium Hyaluronate of lower and higher ranges of molecular weight with low and high concentrations, may provide patients with a more physiologically dynamic HA viscosupplementation and hence a more responsive synovial rheology that improves pain and function in their osteoarthritic knee.

\section{References}

1. Dieppe P. Osteoarthritis. Acta Orthop Scand Suppl 1998;281:2-5.

2. American College of Rheumatology: Recommendations for the medical management of osteoarthritis of the hip and knee: 2000 Update. American College of Rheumatology Subcommittee on osteoarthritis guidelines. Arthritis Rheum 2000;43:1905-15.

3. Balasz EA, Denlinger SL. Viscosupplementation: a new concept in the treatment of osteoarthritis. J Rheumatol 1993;20:3-9.

4. Dahlberg L, Lohmander LS, Ryd L. Intraarticular injections of hyaluronan in patients with cartilage abnormalities and knee pain. Arthritis Rheum 1994;37:521528.

5. Dougados M, Nguyen M, Listrat V, Amor B. High molecular weight sodium hyaluronate (hyalectin) in osteoarthritis of the knee: a 1-year placebo-controlled trial. Osteoarthritis Cartilage 1993;1:97103.

6. Petrella RJ, DiSilvestro MD, Hildebrand C. Effects of hyaluronate sodium on pain and physical functioning in osteoarthritis of the knee. Arch Intern Med 2002;162:292-8.

7. Karlsson J, Sjogren LS, Lohmander LS. Comparison of two hyaluronan drugs and placebo in patients with knee osteoarthritis: a controlled, randomized, double-blind, parallel-design multi-center study. Rheumatology 2002;41:1240-8.

8. Adams ME, Atkinson MH, Lussier AJ et al. The role of viscosupplementation with hylan GF-20 (Synvisc) in the treatment of osteoarthritis of the knee: a Canadian multi-centre trial comparing hylan GF-20 alone, hylan GF-20 with non-steroidal antiinflammatory drugs (NSAIDs) and NSAIDs alone. Osteoarthrits Cartilage 1995;3:21326.

9. Arroll B, Goodyear-Smith F. Corticosteroid injections for osteoarthritis of the knee: meta-analysis. BMJ 2004;328:869.

10. Lo GH, LaValley M, McAlindon T, Felson DT. Intraarticular hyaluronic acid in the treatment of knee osteoarthritis: a meta-analysis. JAMA 2003;290:3115-21.

11. Divine J, Zazulak BT, Hewett TE. Viscosupplementation for Knee Osteo arthritis A Systematic Review. Clin Orth Rel Res 2000;455:113-22.

12. Ghosh P, Guidolin D. Potential mechanism of action of intra-articular hyaluronan therapy in osteoarthritis: are the effects molecular weight dependent? Semin Arthritis Rheum 2002;32:10-37.

13. Roy D. Altman. Intra-articular Sodium Hyaluronate in Osteoarthritis of the Knee. 
Semin Arthritis Rheum 2001;30:11-8.

14. Kotevoglu N, Cakıl P, Hız IO, et al. A prospective randomised controlled clinical trial comparing the efficacy of different molecular weight hyaluronan solutions in the treatment of knee osteoarthritis. Rheumatol Int 2006;26:325-30.

15. Bellamy N, Campbell J, Robinson V, et al. Viscosupplementation for the treatment of osteoarthritis of the knee. Cochrane Database Syst Rev 2006;CD005321.

16. Greenberg DD, Stoker A, Kane S, et al.
Biochemical effects of two different hyaluronic acid products in a co-culture model of osteoarthritis. Osteoarthritis Cartilage 2006;14:814-22.

17. Murakami T, Salai N, Sawae Y, et al. Influence of proteoglycan on time-dependent mechanical behaviors of articular cartilage under constant total compressive deformation. JSME Int J 2004;47:1049-55.

18. Ghosh P, Guidolin D. Potential mechanism of action of intra-articular hyaluronan therapy in osteoarthritis: are the effects of molecular weight dependent? Seminars Arthritis Rheum 2002;32:10-37.

19. Lussier A, Cividino AA, McFarlane CA, et al. Viscosupplementation with hylan for the treatment of osteoarthritis: findings from clinical practice in Canada. $\mathrm{J}$ Rheumatol 1996;23:1579-85.

20 R. J. Petrella. Hyaluronic acid for the treatment of knee osteoarthritis: long-term outcomes from a naturalistic primary care experience. Am J Phys Med Rehabil 2005; 84:8-83. 